\title{
OntAES: Uma Ontologia para Sistemas Adaptativos Educacionais Baseada em Objetos de Aprendizagem e Estilos de Aprendizagem
}

\author{
Vitor C. de Carvalho ${ }^{1}$, Rafael D. Araújo ${ }^{1}$, Hiran N. M. Ferreira ${ }^{2}$ \\ Fabiano A. Dorça ${ }^{1}$, Renan G. Cattelan ${ }^{1}$ \\ ${ }^{1}$ Faculdade de Computação - Universidade Federal de Uberlândia (UFU) \\ Uberlândia, MG, Brasil. \\ ${ }^{2}$ Instituto Federal de Educação, Ciência e Tecnologia do Sul de Minas Gerais \\ (IFSULDEMINAS) - Passos, MG, Brasil \\ \{vitor.carvalho, rafael.araujo\}@ufu.br \\ hiran.ferreira@ifsuldeminas.edu.br, \{renan, fabianodor\}@ufu.br
}

\begin{abstract}
This study aims to present an ontology that supports adaptive systems for education based on a relationship between Learning Objects and a Learning Styles model. The ontology, called OntAES, follows the entire teaching process, from the insertion of OAs and students into the system, to classification and ranking in a personalized and automatic way. In addition, it presents a comparative approach between how to calculate the OA ranking for each student.
\end{abstract}

Resumo. Este estudo tem o objetivo de apresentar uma ontologia que auxilia sistemas adaptivos para educação, baseada num relacionamento entre Objetos de Aprendizagem e em um modelo de Estilos de Aprendizagem. A ontologia, denominada OntAES acompanha todo o processo de ensino, desde a inserção de OAs e estudantes no sistema, até a classificação e ranqueamento de forma personalizada e automática. Além disso, apresenta uma abordagem comparativa entre forma de calcular o ranking de OAs para cada estudante.

\section{Introdução}

O uso de sistemas computacionais é cada vez mais comum no ensino, onde oferecem apoio tanto ao instrutor quanto ao estudante. Esses sistemas utilizam de Objetos de Aprendizagem (OAs) como tecnologia de conteúdo e em muitos casos fornecem personalização e adaptabilidade com o objetivo de adequar o curso à cada aprendiz de forma individual.

Utilizar OAs como objeto de conhecimento é uma abordagem consistente no contexto do ensino, visto que podem ser definidos como quaisquer recursos virtuais de aprendizagem como vídeos, imagens e textos que tenham como objetivo trazer informação a ser assimilada pelo aprendiz. Além disso, os OAs acompanham metadados que os descrevem para garantir a sua acessibilidade, interoperabilidade e a capacidade de serem reusados por diversos sistemas e autores como, por exemplo, o padrão IEEE-LOM [IEEE 2002].

Em geral, esses sistemas possuem algum grau de personalização, o que é necessário, pois, cada estudante vivencia o processo de aprendizagem de forma diferente, demonstrando aspectos cognitivos que dão ênfase em suas melhores habilidades. A 
VI Congresso Brasileiro de Informática na Educação (CBIE 2017)

Anais do XXVIII Simpósio Brasileiro de Informática na Educação (SBIE 2017)

observação desse fenômeno deu origem às pesquisas sobre os Estilos de Aprendizagem (EAs) como o modelo de Felder e Silverman [Felder and Silverman 1988].

Este trabalho traz avanços na construção de uma ontologia aqui denominada como OntAES(Ontology for Adaptive Educational Systems) que representa OAs com metadados no padrão IEEE-LOM relacionados ao modelo de EAs proposto por Felder e Silverman (FSLSM) [Carvalho et al. 2016].

A proposta inclui também uma extensão relativa ao padrão IEEE-LOM para o vocabulário do campo Learning Resource Type, chamado de Customized Learning Experience Online (CLEO) [CLEO 2003], que permite ampliar, através de novos valores para o referido campo, os tipos de recursos de aprendizagem a serem mapeados.

Desta forma, é possível ordenar OAs de acordo com o grau de aderência aos EAs de um estudante específico, o que permite selecionar facilmente um conjunto de OAs mais adequados a cada estudante. Neste contexto, este trabalho apresenta uma análise comparativa de três métodos para ordenação dos OAs.

A abordagem conta então com a evolução de uma ontologia, criada como base o relacionamento existente entre os campos do padrão de metadados IEEE-LOM e CLEO para com os EAs do FSLSM. Experimentos realizados validaram a proposta e mostram que a abordagem é promissora no que tange representar OAs e recomendá-los eficientemente considerando às preferências de aprendizagem do estudante, sendo então uma interessante abordagem de apoio aos sistemas de recomendação de conteúdo de ensino.

O restante deste artigo está organizado da seguinte maneira: na Seção 2, são discutidos trabalhos relacionados; na Seção 3, são apresentados os principais conceitos utilizados na proposta; na Seção 4, é descrita a abordagem proposta e alguns exemplos de sua utilização e resultados; a Seção 5 apresenta um experimento e, por fim, na Seção 6, são feitas as considerações finais.

\section{Trabalhos Relacionados}

O número de pesquisas que aproveitam dos recursos da Web Semântica para educação vem crescendo a cada dia. O trabalho de Rezende et al. 2015, por exemplo, propõe uma Ontologia, a qual nomearam PERSONNA, para o perfil do estudante com objetivo de facilitar a recomendação de conteúdo em sistemas de ensino, utilizando também do contexto em que o aluno se encontra.

Pukkhem 2013 propôs um framework que demonstra como as ontologias podem ser usadas para habilitar o processamento de recursos de aprendizagem por sistemas de recomendação. Nele também são utilizados o FSLSM e o padrão IEEE-LOM de Objetos de Aprendizagem.

Gluz and Vicari 2011 propõem uma Ontologia para representação de Metadados nos padrões IEEE-LOM, DublinCore [Weibel et al. 1998] e o OBAA [Vicari et al. 2010]. Porém, este estudo não oferece mais recursos e demonstrações de uso, deixando em aberto as possíveis funcionalidades existentes.

Temos também trabalhos focados no cálculo realizado ao final do processo de Recomendação, responsável por ranquear os OAs pela forma com que se adequam ao processo de aprendizagem do estudante. Carvalho et al. 2014 e Dorça et al. 2016 seguem 
VI Congresso Brasileiro de Informática na Educação (CBIE 2017)

Anais do XXVIII Simpósio Brasileiro de Informática na Educação (SBIE 2017)

essa linha, onde o primeiro mostra como utilizar cálculos de distância e o segundo utiliza uma fórmula própria, ambos levando em consideração o mesmo mapeamento entre IEEELOM e FSLSM usado neste trabaho.

Este trabalho se destaca trazendo uma evolução à Ontologia criada por [Carvalho et al. 2016] oferecendo ferramentas de tecnologias de inferência, agregando eficácia para os sistemas que necessitam representar seus OAs de forma mais próxima ao modelo do estudante. Assim, é possível realizar recomendação automática e eficente dos OAs de acordo com os EAs do aprendiz.

Também são apresentadas três abordagens de cálculo de ranking de OAs, onde é feita uma comparação que toma os pontos positivos das abordagens apresentadas por Carvalho et al. 2014 e Dorça et al. 2016 para construir uma nova fórmula. Fórmula esta que leva em consideração características do mapeamento entre IEEE-LOM e FSLSM.

\section{Fundamentação Teórica}

Essa seção apresenta conceitos úteis para uma melhor compreensão do trabalho.

\subsection{Modelo de Felder-Silverman}

Estilos de Aprendizagem(EA) dizem respeito às formas cognitivas que os estudantes possuem para melhor assimilar o conceito ensinado. [Graf et al. 2009] cita em seu trabalho que utilização de EAs ajudam a melhorar a eficiencia da adaptatividade dos sistemas de recomendação e trazer modelos mais holísticos.

Entre as teorias de EAs, o modelo criado por Felder and Silverman 1988 (Felder and Silverman Learning Style Model - FSLSM) esta entre os mais utilizados nos trabalhos da área [Graf et al. 2007, Dorça et al. 2013]. Nele, foi identificado que cada estudante tem sua forma de receber e processar informações com eficiência.

O modelo original é composto por quatro dimensões, cada uma possuindo dois EAs, conforme apresentado na Tabela 1. Os indivíduos podem ser representados pela combinação de um estilo para cada uma das quatro dimensões, encaixando em uma num total de 16 diferentes combinações de EA.

\subsection{Padrão IEEE Learning Object Metadata e CLEO}

Todo OA deve possuir importantes atributos, são eles: Interoperabilidade, Acessibilidade e Durabilidade. Interoperabilidade é a capacidade de se usar um OA feito em sistemas diferentes daqueles para qual foram criados. Acessibilidade da a capacidade dos OAs de serem utilizados remotamente e, Durabilidade, que é a possibilidade de utilizar um OA, sem reprojeto ou recriação, mesmo quando a base tecnológica muda.

Para que essas características se mantenham, é necessário que haja um padrão, como o LOM [IEEE 2002]. O LOM é composto por 68 elementos hierarquizados, divididos em 9 categorias, que descrevem a semântica dos metadados de um OA. Neste trabalho, utilizou-se 5 desses elementos, são eles: Structure, Format, Interactivity Type, Learning Resource Type e Interactivity Level.

Structure é o campo que oferece uma descrição da estrutura organizacional do OA. Format nos mostra o tipo de dado trabalhado pelo OA para facilitar a identificação de quais softwares serão necessários no seu acesso. Os tipos permitidos pelo campo 
VI Congresso Brasileiro de Informática na Educação (CBIE 2017)

Anais do XXVIII Simpósio Brasileiro de Informática na Educação (SBIE 2017)

Tabela 1. Tabela de estilos do modelo FSLSM (adaptada de Franzoni et al. 2008).

\begin{tabular}{|c|c|c|}
\hline $\begin{array}{c}\text { Dimensao do Estilo de } \\
\text { Apredizagem }\end{array}$ & Tipo & Descrição \\
\hline \multirow{2}{*}{ Percepção } & Sensitivo (S) & $\begin{array}{l}\text { Sabe lidar com fatos, dados puros e experimentos, é paciente com } \\
\text { os detalhes, mas não gosta de complicações. }\end{array}$ \\
\hline & Intuitivo (I) & $\begin{array}{l}\text { Sabe lidar com principios e teorias, fica facilmente entediado } \\
\text { quando são apresentados muitos detalhes e tende a aceitar } \\
\text { complicações. }\end{array}$ \\
\hline \multirow{2}{*}{ Canal de Entrada } & Visual (Vi) & $\begin{array}{l}\text { Se lembra bem do que viu: imagens, diagramas, tabelas temporais, } \\
\text { filmes, etc. }\end{array}$ \\
\hline & Verbal (Ve) & Se lembra do que escutou, leu ou disse. \\
\hline \multirow{2}{*}{ Processamento } & Ativo (A) & Aprende bem em grupo e com o material que tem em mãos. \\
\hline & Reflexivo (Re) & $\begin{array}{l}\text { Aprende melhor quando pensa e reflete sobre a informação dada a } \\
\text { ele. Trabalha melhor sozinho ou com no máximo mais uma pessoa. }\end{array}$ \\
\hline \multirow{2}{*}{ Entendimento } & Sequencial (Seq) & $\begin{array}{c}\text { Segue um raciocínio linear quando está resolvendo problemas e } \\
\text { pode trabalhar com material especifico uma vez que compreende } \\
\text { ele parcialmente ou superficialmente. }\end{array}$ \\
\hline & Global (G) & $\begin{array}{l}\text { Tem grandes intuiçốes sobre a informação, pode ter dificuldade de } \\
\text { expor como chegou a certo resultado, precisa de uma visão geral. }\end{array}$ \\
\hline
\end{tabular}

Format são definidos pelo registro IANA de tipos MIME(ver RFC2048:1996 [Borenstein and Freed 1996]).

Interactivity Type fornece informação do tipo predominante de aprendizado no OA (Ativo ou Expositivo). Learning Resource Type descreve o tipo específico do OA, como por exemplo: exercício, simulação, questionário, etc. e, por fim, Interactivity Level que informa o nível de interatividade proporcionado pelo OA.

Neste trabalho, também inserimos novos termos ao conjunto de Learning $R e$ source Type do IEEE-LOM. Esses termos, definidos pelo CLEO [CLEO 2003], que suprem a necessidade por representação de OAs que existem, mas não são incluídos no padrão criado. Os novos termos são apresentados na Tabela 2.

Tabela 2. Learning Resource Types adicionados pelo CLEO [CLEO 2003].

\begin{tabular}{|c|c|c|c|c|}
\hline \multicolumn{7}{|c|}{ CLEO Learning Resource Types } \\
\hline additional resource & analogy & assessment & assessment item & attractor \\
\hline attractor & definition & demonstration & example & feedback \\
\hline glossary & guidance & guideline & illustration & importance \\
\hline introduction & non example & note & objective & outline \\
\hline overview & practice & prerequisite & presentation & recall \\
\hline reference & reinforcement & \multicolumn{3}{c}{} \\
\cline { 2 - 3 } & \multicolumn{5}{rl}{}
\end{tabular}

\subsection{Ontologias e Web Semântica}

A parte da web que provê técnicas, tecnologias e padrões que nos permitem atribuir significado às informações presentes é a chamada web semântica. Ela nos traz, por exemplo, importantes tecnologias como o Web Ontology Language [Shadbolt et al. 2006] entre outras dispostas por suas camadas, apresentadas na Figura 1. 


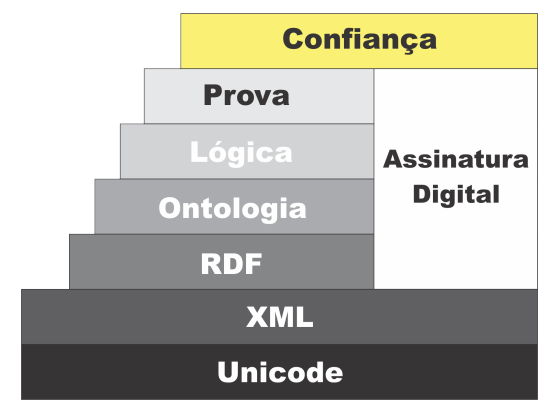

Figura 1. Camadas da Web Semântica. (Adaptado de [Yu 2011])

Dentro da Inteligência Artificial (IA), o conceito de ontologia é conhecido como o conjunto de entidades com suas relações, restrições, axiomas e vocabulário [Freitas 2003]. Dentre os benefícios de se utilizar ontologias, podemos destacar a utilização de uma linguagem declarativa que em geral, envolvem arquivos XML descritas em uma linguagem chamada Web Ontology Language (OWL) [Sant'Anna et al. 2008].

Numa ontologia OWL pode-se incluir: "relações de taxonomia entre classes; propriedades dos tipos de dados e descrições dos atributos de elementos das classes, propriedades do objeto e descrições das relações entre elementos das classes, instâncias das classes e propriedades" [Santos and de Carvalho 2007].

Ontologias são interessantes de serem utilizadas em sistemas que têm como foco o processo de ensino, por auxiliarem na representação do conhecimento de modo formal e completo, além de ser um conceito maduro e possuir ferramentas bem trabalhadas que auxiliam a empregar o conceito. Existem ainda outras vantagens de se utilizar ontologias, destacadas por Freitas 2003.

Com o uso de Ontologias é possível ainda modelar regras em linguagens próprias, como a SWRL [Horrocks et al. 2004] e a SQWRL [O'Connor and Das 2009]. A linguagem SWRL foi elaborada para a criação de regras lógicas de inferência, sendo munida de operadores comuns da lógica proposicional com algumas limitações, como a falta dos operadores "OR"e "NOT".

Já a SQWRL foi criada com a intenção de trazer algumas funções do SQL convencional, tendo então como objetivo, criar queries de pesquisa dentro das ontologias. A SQWRL possui também a capacidade de receber algumas extensões, denominadas builtins, que acrescentam novas funcionalidades à linguagem.

\section{Abordagem Proposta}

Este trabalho propõe uma ontologia, adaptada de Carvalho et al. 2016 que gera uma representação vetorial para cada OA considerando os EAs que atendem, para então, de forma eficiente, recomendar os OAs aos estudantes considerando seus EAs específicos. A Tabela 3 apresenta a base de regras que permite determinar quais EAs satisfazem determinado OA. Cada um dos cinco campos de metadados (Structure, Format, Interactivity Type, Learning Resource Type e Interactivity Level) possuem regras que são mapeadas aos EAs. Por exemplo, se o nível de interatividade do OA é muito alto, então o OA é mais propenso a se enquadrar para estudantes Ativos.

As regras estão descritas em formato Prolog e observando-as, é possível notar que 
VI Congresso Brasileiro de Informática na Educação (CBIE 2017)

Anais do XXVIII Simpósio Brasileiro de Informática na Educação (SBIE 2017)

Tabela 3. Tabela de mapeamento entre os elementos IEEE-LOM para os Estilos de Aprendizagem Felder-Silverman.

\begin{tabular}{|c|c|c|c|c|c|}
\hline Structure & Format & $\begin{array}{c}\text { Interactivity Type } \\
\text { (IT) }\end{array}$ & \multicolumn{2}{|c|}{$\begin{array}{c}\text { Learning Resource Type } \\
\text { (LRT) + CLEO }\end{array}$} & $\begin{array}{l}\text { Interactivity Level } \\
\text { (IL) }\end{array}$ \\
\hline $\begin{array}{l}\text { 1:Sequential(X):- } \\
\text { Structure(X,"atomic"); } \\
\text { Structure(X,"linear"). } \\
\text { 2:Global(X):- } \\
\text { Structure(X,"collection"); } \\
\text { Structure(X,"networked"); } \\
\text { Structure(X,"hierarchical"). }\end{array}$ & \begin{tabular}{|l} 
3:Visual(X):- \\
Format(X,"video"); \\
Format(X,"image"). \\
4:Verbal(X):- \\
Format:(X,"text"); \\
Format(X,"audio"). \\
5:Active(X):- \\
Format(X,"application"). \\
6:Reflective(X):- \\
Format(X,"video"); \\
Format (X,"audio"); \\
Format(X,"image").
\end{tabular} & \begin{tabular}{|l} 
7:Active(X):- \\
IT(X,"active"); \\
IT(X,"mixed"). \\
8:Reflective(X):- \\
IT(X,"expositive"); \\
IT(X,"mixed").
\end{tabular} & \begin{tabular}{|l} 
9:Active(X):- \\
LRT(X,"exercise"); \\
LRT(X,"simulation"); \\
LRT(X,"questionnaire"); \\
LRT(X,"exam"); \\
LRT(X,"experiment"); \\
LRT(X,"problem statem."); \\
LRT(X,"self assessment"); \\
LRT(X,"demonstration"). \\
10:Reflective(X):- \\
LRT(X,"diagram"); \\
LRT(X,"figure"); \\
LRT(X,"graph"); \\
LRTX,"index"); \\
LRT(X,"slide"); \\
LRT(X,"table"); \\
LRT(X,"narrative text"); \\
LRT(X,"lecture"); \\
LRT(X,"definition"). \\
11:Sensitive(X):- \\
LRT(X,"simulation"); \\
LRT(X,"figure"); \\
LRT(X,"graph"); \\
LRT(X,"index"); \\
LRT(X,"table"); \\
LRT(X,"experiment"); \\
LRT(X,"assessment"). \\
12:Intuitive(X):- \\
LRT(X,"diagram"); \\
LRT(X,"attractor"); \\
LRT(X,"example"); \\
LRT(X,"objective").
\end{tabular} & \begin{tabular}{|l} 
13:Visual(X):- \\
LRT(X,"diagram"); \\
LRT(X,"figure"); \\
LRT(X,"graph"); \\
LRT(X,"experiment"); \\
LRT(X,"attractor"). \\
14:Verbal(X):- \\
LRT(X,"exercise"); \\
LRT(X,"questionnaire"); \\
LRT(X,"index"); \\
LRT(X,"table"); \\
LRT(X,"narrative text"); \\
LRT(X,"exam"); \\
LRT(X,"problem statem."); \\
LRT(X,"self assessment"); \\
LRT(X,"lecture"); \\
LRT(X,"definition"). \\
15: Global(X):- \\
LRT(X,"definition"); \\
LRT(X,"example"); \\
LRT(X,"introduction"); \\
LRT(X,"outline"); \\
LRT(X,"overview"); \\
LRT(X,"summary"). \\
16: Sequential(X):- \\
LRT(X,"community"); \\
LRT(X,"demonstration"); \\
LRT(X,"objective").
\end{tabular} & \begin{tabular}{|l} 
17:Active(X):- \\
IL(X,"medium"); \\
IL(X,"high"); \\
IL(X,"very high"). \\
18:Reflective(X):- \\
IL(X,"very low"); \\
IL(X,"low"); \\
IL(X,"medium").
\end{tabular} \\
\hline
\end{tabular}

cada valor de um campo de determinado OA, representado pela variável X, é relacionado a um EA do modelo FSLSM.Essa base de regras foi anteriormente proposta em [Dorça et al. 2016], porém, acrescida de um subconjunto do vocabulário CLEO para o campo Learning Resource Type, que também possuem valores passíveis de serem mapeados para o FSLSM, conforme mostrado pela Tabela 4.

Tabela 4. Vocabulário do CLEO acrescentado à base de regras.

\begin{tabular}{|c|c|c|c|c|c|}
\hline additional resource & assessment & attractor & community & definition & demonstration \\
\hline example & introduction & objective & outline & overview & summary \\
\hline
\end{tabular}

A partir da base de regras criadas, foi criada uma ontologia que possui duas classes. A primeira classe modela os OAs e a segunda classe modela os EAs, conforme apresentado na Figura 2. A classe OA possui cinco subclasses representando as 5 categorias do padrão utilizadas no mapeamento. Cada subclasse é composta por indivíduos que representam os valores que cada campo pode receber de acordo com o padrão IEEE-LOM. Já a classe EA é composta apenas por indivíduos, onde cada indivíduo representa um dos oito EAs de Felder. Além disso, foram criados dois Object Property para a Ontologia, para relacionar os conceitos inclusos: o has e o is [Carvalho et al. 2016].

A ontologia, inicialmente desenvolvida no trabalho Carvalho et al. 2016 foi refeita e revalidada, através da metodologia 101 [Noy and McGuinness 2001]. Os regras e formato utilizados foram os mesmos descritos no já citado trabalho, acrescidos de regras referentes aos termos CLEO adicionados à base de regras.

A ontologia desenvolvida foi denominada OntAES (Ontology for Adaptive Educational Systems). Regras de inferência criadas na ontologia através de linguagens como a SWRL [Horrocks et al. 2004] e a SQWRL [O'Connor and Das 2009] criam uma 
Figura 2. Representação gráfica da ontologia criada [Carvalho et al. 2016].

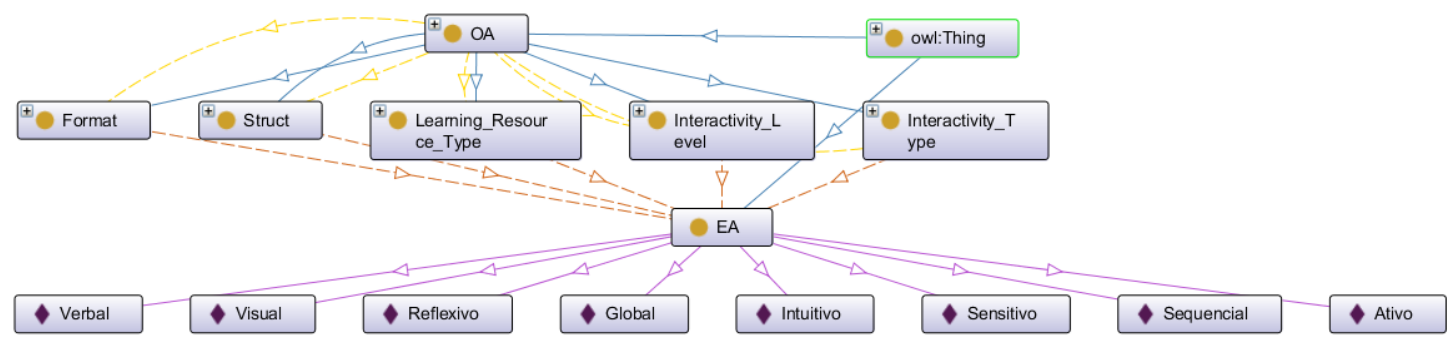

representação vetorial de 8 dimensões (uma para cada EA do FSLSM) dos OAs para indicar a relação dos OAs com intuito de representar matematicamente o quão aderente é um OA em relação aos EAs.

\section{Experimento}

Os experimentos foram realizados com a base de dados do Classroom eXperience [Ferreira et al. 2012], um ambiente educacional ubíquo desenvolvido para capturar aulas e armazená-los como OAs. Desta forma, foi possível conhecer a abordagem mais eficiente para recomendação de OAs considerando os resultados gerados pela ontologia proposta.

A fim de apresentação, usaremos o mesmo exemplo dado por [Carvalho et al. 2016], apresentado pela Tabela 5. Nela, pode-se observar os atributos do OA que são apresentados na segunda coluna e, na primeira coluna, como são inseridos na ontologia.

Tabela 5. Exemplo de OA na Ontologia [Carvalho et al. 2016].

\begin{tabular}{ll}
\hline Atributos & Campos IEEE-LOM \\
\hline has(OA1, mixed) & Interactivity Type \\
has(OA1, collection) & Struct \\
has(OA1, video) & Format \\
has(OA1, table) & Learning Resource Type \\
has(OA1, medium) & Interactivity Level \\
\hline
\end{tabular}

A Tabela 6 apresenta o resultado das inferências realizadas pela ontologia para o OA mostrado, onde a primeira coluna referencia os atributos do OA incluídos na ontologia e a segunda os relacionamentos resultantes. E, finalmente, a Tabela 7 apresenta a vetorização do OA apresentado na Tabela 5 [Carvalho et al. 2016], cujos valores representam a quantidade de regras atendidas pelo OA.

Com o resultado obtido pela etapa final do processo de vetorização de OAs, entramos então no processo de recomendação, que consiste em ranquear os OAs por similaridade. Aqui, várias técnicas podem ser aplicadas, foram comparadas então três delas. Para que as fórmulas funcionem, os estudantes devem ser modelados utilizando o FSLSM de forma probabilística, como apresentado em [Dorça et al. 2016].

A Equação (1), proposta em [Dorça et al. 2016], mostra uma forma simples de se calcular a relevância, onde $Q_{i}$, é a quantidade de regras satisfeitas para o EA $i$ da representação vetorial do OA e $E_{i}$ é a probabilidade do estudante preferir OAs do EA $i$. 
VI Congresso Brasileiro de Informática na Educação (CBIE 2017)

Anais do XXVIII Simpósio Brasileiro de Informática na Educação (SBIE 2017)

Tabela 6. Resultado das inferências da Ontologia [Carvalho et al. 2016].

\begin{tabular}{|l|l|}
\hline Conceito de OA & Resultado da inferência \\
\hline has(OA1, mixed) & is(mixed, Ativo) \\
\hline has(OA1, collection) & is(collection, Global) \\
\hline has(OA1, video) & is(video, Visual) \\
\hline has(OA1, table) & is(video, Reflexivo) \\
\hline has(OA1, medium) & is(table, Verbal) \\
\hline & is(table, Reflexivo) \\
\hline
\end{tabular}

Tabela 7. OA1 Vetorizado [Carvalho et al. 2016].

\begin{tabular}{cccccccc}
\hline \multicolumn{8}{c}{ OA1 } \\
\hline Ativo & Reflexivo & Visual & Verbal & Sequencial & Global & Intuitivo & Sensitivo \\
2 & 4 & 1 & 1 & 0 & 1 & 0 & 1 \\
\hline
\end{tabular}

Já a Equação (2), apresenta a fórmula proposta por Carvalho et al. 2014, onde, considerando um Estudante E, a relevância de um OA é calculado como a distância de Manhattan entre o modelo do estudante e este OA. Aqui, porém, considera-se a proporção de um EA para o outro dentro de uma mesma dimensão. Assim, o OA vetorizado na Tabela 7 seria convertido para os valores apresentados na Tabela 8.

\section{Tabela 8. OA1 vetorizado convertido para proporções entre os EAs de mesma} dimensão.

\begin{tabular}{cccccccc}
\hline \multicolumn{1}{c}{ OA1 } \\
\hline Ativo & Reflexivo & Visual & Verbal & Sequencial & Global & Intuitivo & Sensitivo \\
33 & 66 & 50 & 50 & 0 & 100 & 0 & 100 \\
\hline
\end{tabular}

A Equação (2) teve melhor resultado pois leva em consideração as diferenças de número de regras existentes para cada EA. Porém, ainda não é considerada a diferença de número regras entre as dimensões do FSLSM em nossa base. Assim, surge a Equação (3), onde o valor de $P_{i}$ é o cálculo de proporção da quantidade de regras atendidas por aquele EA, em comparação às outras dimensões.

$$
\sum_{i=1}^{8}\left(Q_{i} \times E_{i}\right) \quad(1) \quad \sum_{i=1}^{8}\left|O A_{i}-E_{i}\right| \quad \text { (2) } \quad \sum_{i=1}^{8}\left(P_{i} \times E_{i}\right)
$$

\section{Considerações Finais}

Este trabalho apresentou a evolução de uma ontologia (OntAES) já apresentada anteriormente que modela a relação entre OAs e EAs, de forma a oferecer suporte aos pro- 
VI Congresso Brasileiro de Informática na Educação (CBIE 2017)

Anais do XXVIII Simpósio Brasileiro de Informática na Educação (SBIE 2017)

cessos de recomendação personalizada de conteúdo. A aplicação da OntAES em sistemas de recomendação permite facilitar o processo de ranqueamento, apresentando uma vetorização dos OAs que os aproxima da apresentação de EAs de forma probabilística.

A ontologia foi validada a partir de experimentos, e resultados promissores foram obtidos, demonstrando o potencial de contribuição desta proposta às abordagens para recomendação personalizada de conteúdo em sistemas educacionais. Além disso, através dos experimentos, foi obtida uma melhor forma de calcular os rankings entre OAs e Estudantes, que leva em consideração características do mapeamento apresentado, que trabalhos anteriores não visitaram.

\section{Agradecimentos}

Os autores agradecem as agências brasileiras de pesquisa CNPq, CAPES, FAPEMIG e a PROPP/UFU pelo apoio concedido a este trabalho.

\section{Referências}

Borenstein, N. S. and Freed, N. (1996). Multipurpose internet mail extensions (mime) part one: Format of internet message bodies.

Carvalho, V., Mendes, M., Ferreira, H., Dorça, F., and Cattelan, R. (2016). Uma ontologia para apoio à recomendação automática e personalizada de conteúdo considerando estilos de aprendizagem de estudantes em sistemas adaptativos para educação. In $\mathrm{Bra}$ zilian Symposium on Computers in Education (Simpósio Brasileiro de Informática na Educação-SBIE), volume 27, page 1175.

Carvalho, V. C., Dorça, F. A., Cattelan, R. G., and Araújo, R. D. (2014). Uma Abordagem para Recomendação Automática e Dinâmica de Objetos de Aprendizagem Baseada em Estilos de Aprendizagem. In Anais do Simpósio Brasileiro de Informática na Educação, volume 25, page 1188 .

CLEO (2003). CLEO Extensions to the IEEE Learning Object Metadata. Technical report, CLEO Collaborative Partners (Cisco Systems, Inc., IBM Corporation, Microsoft Corporation, Thomson NETg). Available on https: //www.oasis-open.org/ committees/download.php/20490/CLEO\_LOM\_Ext\_v1d1a.pdf.

Accessed in: October, 2016.

Dorça, F. A., Araújo, R. D., De Carvalho, V. C., Resende, D. T., and Cattelan, R. G. (2016). An Automatic and Dynamic Approach for Personalized Recommendation of Learning Objects Considering Students Learning Styles: An Experimental Analysis. Informatics in Education, 15(1):45-62.

Dorça, F. A., Lima, L. V., Fernandes, M. A., and Lopes, C. R. (2013). A New Approach to Discover Students Learning Styles in Adaptive Educational Systems. Revista Brasileira de Informática na Educação, 21(1):25-2013.

Felder, R. M. and Silverman, L. K. (1988). Learning and teaching styles in engineering education. Engineering education, 78(7):674-681.

Ferreira, H. N. M., Araujo, R. D., de Amo, S. A., and Cattelan, R. G. (2012). Classroom Experience: A Platform for Multimedia Capture and Access in Instrumented Educational Environments. In 2012 Brazilian Symposium on Collaborative Systems, pages 59-64. IEEE. 
VI Congresso Brasileiro de Informática na Educação (CBIE 2017)

Anais do XXVIII Simpósio Brasileiro de Informática na Educação (SBIE 2017)

Franzoni, A. L., Assar, S., Defude, B., and Rojas, J. (2008). Student learning styles adaptation method based on teaching strategies and electronic media. In Advanced Learning Technologies, 2008. ICALT'08. Eighth IEEE International Conference on, pages 778-782. IEEE.

Freitas, F. L. G. (2003). Ontologias ea web semântica. Sociedade Brasileira de Computação (SBC). II Jornada de Mini-Cursos de Inteligência Artificial, pages 1-52.

Gluz, J. C. and Vicari, R. M. (2011). Uma Ontologia OWL para Metadados IEEELOM, DublinCore e OBAA. Anais do Simpósio Brasileiro de Informática na Educação, 1(1).

Graf, S., Liu, T.-C., Kinshuk, Chen, N.-S., and Yang, S. J. (2009). Learning styles and cognitive traits - Their relationship and its benefits in web-based educational systems. Computers in Human Behavior, 25(6):1280-1289.

Graf, S., Viola, S. R., Leo, T., and Kinshuk (2007). In-depth analysis of the feldersilverman learning style dimensions. Journal of Research on Technology in Education, 40(1):79-93.

Horrocks, I., Patel-Schneider, P. F., Boley, H., Tabet, S., Grosof, B., Dean, M., et al. (2004). Swrl: A semantic web rule language combining owl and ruleml. W3C Member submission, 21:79.

IEEE (2002). 1484.12.1-2002 IEEE Standard for Learning Object Metadata.

Noy, N. F. and McGuinness, D. L. (2001). Ontology Development 101: A Guide to Creating Your First Ontology.

O'Connor, M. J. and Das, A. K. (2009). Sqwrl: A query language for owl. In OWLED, volume 529.

Pukkhem, N. (2013). Ontology-based Semantic Approach for Learning Object Recommendation. ACEEE Int. J. on Information Technology, 3(4).

Rezende, P. A. A., Pereira, C. K., Campos, F., David, J. M. N., and Braga, R. (2015). PERSONNA: proposta de ontologia de contexto e perfil de alunos para recomendação de objetos de aprendizagem. Revista Brasileira de Informática na Educação, 23(01):70.

Sant'Anna, F., Cerqueira, R., and Soares, L. F. G. (2008). Nclua: objetos imperativos lua na linguagem declarativa ncl. In Proceedings of the 14th Brazilian Symposium on Multimedia and the Web, pages 83-90. ACM.

Santos, F. C. and de Carvalho, C. L. (2007). Aplicações de suporte à web semântica.

Shadbolt, N., Hall, W., and Berners-Lee, T. (2006). The semantic web revisited. Intelligent Systems, IEEE, 21(3):96-101.

Vicari, R. M., Bez, M., da Silva, J. M. C., Ribeiro, A., Gluz, J. C., Passerino, L., Santos, E., Primo, T., Rossi, L., Bordignon, A., et al. (2010). Proposta brasileira de metadados para objetos de aprendizagem baseados em agentes (obaa).

Weibel, S., Kunze, J., Lagoze, C., and Wolf, M. (1998). Dublin core metadata for resource discovery. Technical report.

Yu, L. (2011). A developer's guide to the semantic web. Springer. 